\title{
Téoros
}

Revue de recherche en tourisme

\section{Un « New-Deal » touristique européen ?}

\section{Jean-Paul De Smedt}

Volume 8, numéro 1, mars 1989

France-Québec

URI : https://id.erudit.org/iderudit/1080349ar

DOI : https://doi.org/10.7202/1080349ar

Aller au sommaire du numéro

\section{Éditeur(s)}

Université du Québec à Montréal

\section{ISSN}

0712-8657 (imprimé)

1923-2705 (numérique)

Découvrir la revue

\section{Citer cet article}

De Smedt, J.-P. (1989). Un " New-Deal » touristique européen ? Téoros, 8(1), 8-9. https://doi.org/10.7202/1080349ar d'utilisation que vous pouvez consulter en ligne.

https://apropos.erudit.org/fr/usagers/politique-dutilisation/ 


\section{Un "New-Deal" touristique européen?}

Dans le cadre de la Bourse Touristique de Lisbonne, un colloque sur le theme 'le tourisme en progres." s'est tenu le 12 janvier dernier. Les communications de plusieurs personnalités "touristiques' "étrangères des États-Unis, de Grande-Bretagne, d'Espagne, du Brésil, de Belgique et de France ont permis de se faire une ides sur les centres d'interêt de chacun à une époque où (surtout en Europe) de grandes mutations se préparent.

L'ouverture européenne de 93, si elle n'était pas précisément à l'ordre du jour, constituait la toile de fond des échanges, ce qui n'était pas contradictoire avec les intentions du pays organisateur, lui-même membre A part entière de la CEE, dont il attend beaucoup en matière de tourisme. Il est vrai qu'avec la Grèce, le Portugal est bénéficiaire de fonds européens $\mathrm{d}^{4}$ aide au développement du tourisme.
Dans une Europe touristique, formée de plusieurs groupes de pays ayant chacun sa spécialisation, la perception de 93 est quelque peu différente entre un "Nord" émetteur, un "Sud" récepteur et la France qui occupe une situation particulière.

Pour le groupe des pays de l'Europe du nord, 1992 est d'abord une grande confrontation entre pays émetteurs avec tour-operators, distributeurs et compagnies aériennes en première ligne. $\tilde{A}$ chacun la meilleure stratégie pour conquérir de nouveaux marchés, de nouvelles positions (en particulier le marché français). Sur ce gigantesque échiquier de plus de 300 millions de consommateurs chacun se place en vue des grands coups de maître. Pour l'instant ce sont surtout les pions qui bougent, les grosses pièces restent sur des positions stratégiques plutôt défensives. $\mathrm{La}$ confidentialité est de rigueur. Les incertitudes dominent toujours, en particulier celles liées aux stratégies des compagnies aériennes, ce qui rend impossible la définition de scénarios crédibles sur les stratégies, la nouvelle donne de l'operating et de la distribution. Selon toute vraisemblance, et cette opinion alimentait bien des discussions de couloirs, les (grands) tour-operators généralistes français, belges ou autres, qui sont des "moyens europeens" risquent de faire les frais de l'opération. Trop petits pour prétendre à une place dans la compétitivité européenne, trop grands pour une clientèle française, qui reste individualiste et trouve encore chez elle de quoi satisfaire ses besoins de détente et de loisirs (l'offre française ne profite-t-elle pas aussi aussi des efforts faits pour accueillir les étrangers?), ils n'auront à terme de salut que dans leur affiliation avec un tour-operating europeen (pur beurre) affiliation apparemment déjâ engagée décidé à conquérir quelques parts du marché français émetteur, qui reste désespérément en-dessous des normes anglo-saxonnes.

La confrontation à aussi une dimension règlementaire: elle se polarise sur la nature du contrat de voyage à forfait ${ }^{(1)}$ qui se discute a Bruxelles avec, comme point d'achoppement la définition des responsabilités du distributeur: sera-t-il entièrement responsable vis-à-vis de son client du respect du contrat de voyage? Derrière une réglementation qui se prétend "protectrice du consommateur", les catalogues vont-ils encore longtemps prévoir des entorses aux prestations prévues dans le contrat de vente?
Un débat épique s'est instauré entre un responsable d'un Tour-operating belge pour qui cette disposition est excessive, et un Anglais pour qui le consommateur doit (enfin) être roi et ne plus subir les désagréments de prestataires qui se rejettent mutuellement les responsabilites des points litigieux. De multiples exemples d'aventures survenues à des touristes anglais ont mis en relief l'attitude de distributeurs ne respectant pas les dispositions du contrat de voyage. La position "consumériste" anglaise, très argumentée, si elle ne sort pas vainqueur des débats de Bruxelles, devrait marquer de son sceau les futures dispositions. Une telle réglementation ne va-t-elle pas favoriser les distributeurs qui contrólent la production des services aux consommateurs, ceux qui sont les mieux organisés, qui ont les moyens de garantir les produits qu'ils vendent, c'est â dire les plus forts, ayant procédé à des intégrations verticales? Ne va-t-elle pas prendre à contre pieds nos milliers de petits distributeurs français (indépendants) qui, pour la plupart, ne connaissent pas ou connaissent mal ce qu'ils vendent? A première vue, les agents de voyages français ne semblent pas particulierement préoccupés par ce qui se trame à Bruxelles. Au Pousada, je demandais à un "snavisfe $^{72}$ de base son sentiment sur ce problème épineux. J'obtenais en général des réponses évasives, mon interlocuteur gêné finissait par accuser Mr Stirn de ne pas être un Ministre à la hauteur et le débat en restait là. Décidément, depuis 89 couper les têtes reste notre b.a.ba stratégique... Les pays de l'Europe du sud sont engagés à fond dans le réceptif touristique, ils jouent de plus en plus fort, peut-être au-delà du raisonnable, la carte du tourisme comme industrie susceptible de générer développement économique et devises. Cela ne va pas sans difficultés et j'ai assisté à un débat portugoportugais sur le développement touristique et le développement régional. Un représentant du pouvoir central et un investisseur se sont cordialement expliques sur l'inexploitation de potentialités régionales... sur la nature de l'intervention de l'État: tourisme partout? ou pôles touristiques? Bref, le difficile équilibre à trouver entre une redistribution de crédits égalitariste (le saupoudrage) et la recherche d'une efficacité économique, n'est pas l'apanage de l'hexagone. Je me suis cru, un instant, au coeur du Massif Central...

A propos d'équilibre, le Portugal connait une difficile gestation. Saisonnalité, taux de saturation en haute saison d'été, tourisme haut 
de gamme, sont les maitres mots des responsables du développement touristique portugais. L'un d'eux, responsable de l'Algarve, région portugaise des plus touristiques, transformee en "colonie anglaise" avec ses pubs, ses quartiers, jusqu'aux boites aux lettres, qui sont la réplique exacte de la perfide Albion.... me confiait ses soucis de diversification de clientèles. II vient de faire la douloureuse expérience de la perversité d'une mono-clientèle: à la suite d'un accident ayant entrainé la mort de deux sujets de sa majesté, la presse anglaise lui a fait perdre 50.000 séjours...

Entre pays récepteurs la concurrence est vive. Espagne, Portugal, pays du pourtour méditerranéen, tous se livrent à des investissements massifs pour du réceptif touristique. L'atout de ces pays du sud restent leur attrait hors Europe et un Américain, à l'esprit on ne peut plus pragmatique de cow-boy, a donné à l'assistance une véritable leçon sur l'art et la manière d'aller à la conquête de l'ouest. Au grand dam des présents, voilà que ce personnage à la Hemigway nous apprend que dans le pays, le plus libre au monde, seulement $10 \%$ des Américains sont possesseurs d'un passeport. Une pièce pourtant indispensable pour aller en Europe. Selon cet intervenant, le premier frein au marché américain est une... formalité administrative, qui réduit le nombre d'Américains du nord suceptibles de venir en Europe de 200 à 20 millions... Il suggère aux distributeurs de produits portugais de prendre en charge une partie du coût du passeport, une manière de promouvoir le Portugal aux USA. Notre Maison de la France ${ }^{(3)}$ y-a-telle pensé?

\section{Les pays de \\ I'europe du centre...}

L'originalité de ces pays se trouve dans cette situation mi-récepteur, mi-émetteur qui les singularisent des pays à forts bataillons de voyageurs aे forfait (pays de l'Europe du nord) et des pays faiblement émetteurs, ayant un réceptif massif (pays de l'Europe du sud). Deux intervenants ${ }^{4 \mid}$ ont mis en avant l'originalité française, qui découle de cette situation mi-chèvre mi-chou. L'un a mis l'accent sur "les produits touristiques alternatifs", mot barbare pour désigner les produits porteurs d'animation de sejour, ou de loisirs (golf, tourisme de santé, sportifs, ex: le polo) qui complètent l'offre d'hébergement traditionnelle. Il est à remarquer l'intérêt suscité par ce type de développement qui contraste avec le modele immobilier et d'animation $35^{5}$ littoral toujours dominant dans l'offre bas de gamme de l'Europe du sud. Un intervenant français a fait un véritable "tabac" avec sa présentation de notre système de formation continue. Voir nos voisins plébisciter notre formation était un peu surprenant pour des Français qui n'ont de cesse de la mettre en cause... Dans une Europe touristique en mutation, avec une complémentarité qui s"affirme (le Nord émetteur, le Sud récepteur) et qui risquent demain d'institu- tionnaliser la répartition européenne du travail touristique, la France apparait quelque peu isolée, dans une situation inédite, hors des modèles dominants, tant émetteurs que récepteurs, impossibles à reproduire intégralement chez elle. Quelle région littorale francaise "supporterait" "les niveaux de frequentation de l'Algarve ou d'Estoril? Entre un réceptif territorial replié et un tour-operating complètement tourné vers l'extérieur, on retrouve dans "l'industrie touristique française "la même fracture, une sorte de modèle réduit européen, avec cette différence majeure qu'en France ces deux mondes s'ignorent superbement. Les relations s'établissent entre émetteurs français/récepteurs étrangers et inversement, entre récepteurs francais/émetteurs étrangers.

En tourisme, comme en géographie, la France bénéficie d'une position centrale qu'on retrouve dans l'attitude de ses acteurs touristiques. Pour certains, l'industrie touristique française n'a d'avenir que dans le développement du tour-operating. Pour $d^{\prime}$ autres, ne compte que l'offre nationale, chacun reprenant à son compte, selon ses intérêts, le modèle européen du nord ou du sud. La voie médiane française est interprêtée comme une faiblesse, tant vis-ă-vis de la puissance distributrice des pays du nord que vis-ă-vis du réceptif massif du sud. Ne reproche-t-on pas à la fois à l'Industrie Touristique Française de ne pas avoir de touroperating et de distribution à l'anglaise ou à l'allemande et en même temps d'être, en receptif, dépassée par l'Espagne. Pouvonsnous etre les premiers partout? La croyance, quasi religieuse, d'une possibilité de marché français du tour-operating identique aux pays du nord fait fi des habitudes françaises. On retrouve là, le même discours que celui que l'on nous tenait à propos des parcs à thème. Certains, qui croyaient que les habitudes françaises allaient se transformer par la magie de l'offre, assistent à la liquidation des biens de Zygofolis ${ }^{(6)}$.

Le tourisme connait aussi ces phênomènes de mode. Une façon de prendre ses rêves pour des réalités. S'agissant du développement du tourisme on est particulierement servi. Ceux qui ne voient lè développement du tourisme européen qu'à travers le développpement du tour-operating ne risquent-ils pas d'avoir les mêmes déceptions que ceux qui voulaient nous vendre du loisir aseptisé? On me pardonnera cette infamie, mais je me demande si ce n'est pas le contraire qui risque de se passer avec l'élargissement de l'Europe. L'individualisation de la consommation touristique à la française - apparaissant possible aux consommateurs du nord et devenue économiquement possible pour ceux du sud, dont le marché émetteur progresse - ne risque-t-elle pas de faire tâche d'huile dans une Europe unifice. L'harmonisation progressive des réglementations, des normes, la transparence des relations de service, le développement des échanges, des voies de communication, la pratique généralisée des langues, la création d'une monnaie com- mune, etc. devraient sécuriser le consommateur européen lamda, le libérer des contraintes qui l'amènent à se réfugier dans la sécurisation du voyage à forfait.

Le tour-operating européen ne perdra-t-il pas progressivement sa fonction d'interface protectrice du consommateur du nord qui se méfie, et pour cause, des Latins pas toujours très sérieux sur la qualité des prestations et des prix?

Ce touriste européen du futur ne rechercherat-il pas, pour ses sejours dans les provinces du vieux continent, la qualité d'une offre qui risque de mal s' accommoder d'une standardisation touristique industrielle excessive, que la guerre des prix et les dérèglementations provoqueront inévitablement?

Les opinions publiques des grandes régions réceptrices du sud de l'Europe accepteronst-elles encore longtemps les grandes invasions, destructrices des équilibres écologiques auxquelles ne répond mêtme pas l'assurance des revenus touristiques stables?

Les grandes migrations "bas de gamme" que connait encore le sud de l'Europe ne vontelles pas se déplacer ailleurs, trouver refuge dans ces pays, ou, comme pour les biens industriels, les coûts de production ne sont plus "concurrentiels"?

Dans ces conditions, si le nord de l'Europe reste (et sans doute devient encore plus) émetteur, le sud ne va-t-il pas, lui, changer sa fonction réceptrice? la France ne préfigure-t-elle pas ce que sera demain la spécialisation touristique sud Européenne?

Le réceptif français prétend souffrir d'un manque de structures de commercialisation. La présence insuffisante des destinations françaises dans les catalogues des T.O. britanniques, allemands et autres, est-t-elle dûe à un volontarisme commercial déficient ou à la réalité de la demande étrangère? La tendance à voir 1993 comme un danger le tourisme français à partir de la concentration du tour-operating européen, n'est-elle pas excessive au regard de ce qu'il représente et (représentera) dans la commercialisation de l'offre française.

\section{Notes}

(11) Cf article de Lucien Rapp pour une réglementation europeenne du contrat de vovage. Cahier d'ESpacES No 13 - Fturier 89

121 Adherant au S.N.A.V., Syndicat National des Agants de Voyage qui "contralent" la profession et bénéficient de ce fait d'une situation de monopole.

|3| Institution chargé de promouwoir la "destination France" à l'átranger.

141 II s'agit de Vincent Bruneas et de Serge Perrot,

(5) 3S: See, Suñ. Sex

(6) Parc d'attractions situe à Nice en liquidation judiciaire:- traduction d'un kchec retentissant des parcs d’attractions en France: 